J. Michael Henderson, B.S., M.B., B.Ch.

Caracciola, Nuvolari, Fangio, Moss, Clark; since the dawn of motor racing, each era has had its heroes - men such as these, whose exceptional skill has been recognised by their competitors and supporters alike. What is it that sets these men apart? Can we say, each year, that the 'top twenty' are indeed the finest racing drivers in the world? As sportsmen, racing drivers are unique in the combination of mental and physical stress to which they are subjected. Who do some, apparently gifted, never succeed in Formula One racing? Just what is so different physiologically and psychologically, about the top class racing driver?

Basically, he is faced by a clearly defined task. In competition with other drivers in similar vehicles, his aim is to control a racing car around a closed circuit with all possible speed and avoiding errors. In so doing he works as an extremely adaptable computing system, analysing information and feeding signals to the car as part of the whole man/machine complex. The efficiency with which he performs this task defines his ability as a recing driver, and is closely related to his mental and physical make-up, his relationship with the car, and on the task itself.

\title{
The Man
}

Psychologically, racing drivers of the top class have been shown to form a clearly distinguishable group; predictably, the more successful the driver the more he conforms to the pattern of this group. The successful racing driver is a high achievement individual and would tend to succeed in many other activities, but successful men from other sporting and academic fields have clearly different personality patterns. The successful driver is an independent man who relies on himself rather than on other people, whom he tends to dominate. Contrary to popular opinion he tends to be reserved, with little need to be part of a group despite high leadership potential. He has great capacity for thinking in abstract terms, one of the factors which determines his above average intelligence. But what shows above all is a burning spirit of competition, indicated by a continual need to surpass his own past performance and the performance of others, and to grapple with any problems presented by the environment. In contrast, less successful drivers score badly in psychological tests which determine the above personality factors. Retrospective surveys have shown a correlation of personality and racing safety, whereas attempts to relate physiological factors - such as reaction time and vision - to a history of racing success have been less successful.

Physically, there appears to be nothing out of the ordinary in the successful driver. Men of this calibre tend to maintain a high standard of physical fitness, which will aid their ability to perform well under stress. Studies among groups of aircraft pilots have shown no relationship between somatotype and operator performance, and the same result 
would be expected if we tried to relate racing driver physique with skill. That many top drivers are small men simply demonstrates that a $301 \mathrm{~b}$ weight difference is worth a lot of bhp.

Physiologically, leading racing drivers will be well equipped; but there is no evidence that they posess superhuman powers, merely welldeveloped body systems which are used to full capacity. In particular, the body's sensory organs, special senses and neuro-muscular systems will be called upon to function as part of the computing mechanism which makes up the man who controls the racing car. Inasmuch as the driver can be likened to a computer, we are concered with input to and output from that mechanism. Forces emanating in the racing car will be received by the sensory organs - the input system - and in response to these forces the driver will use his neuromuscular apparatus - the output - to control the car. From the seat, steering wheel and pedals flows information regarding the movement and position of car and controls, which is accepted by touch and pressure receptors in the skin, deep tissues and joints. These kinaesthetic cues are transmitted to the brain as part of the whole sensory input, to be related to visual information as to the car's position on the track, aural information giving instant guidance on engine speed. Concurrently, the vestibular organs respond to accelerative forces, both radial and linear; the efficiency with which they do this, and the speed with which the body reacts, must go a long way to determining the ultimate ability of the racing drive.

In ergonomic terms, the sensory mechanisms must be given a full chance of collecting the relevant stimuli. So, the driving seat should be all-embracing and but thinly padded; similarly, the overals and driving shoes should be thin and flexible. Pedals should swing in an arc through the natural centre position of the ankle joint. The straight arm driving style which has become associated with light and direct steering via a tiny wheel means that most of the fine, faster signals transmitted through the steering mechanism will be picked up by the sensitive fingers and wrists rather than by the relatively insensitive arms and shoulders. First class all round vision gives the eyes maximum opportunity to accept all available visual information, and intelligent siting of the instruments will mean that the eyes will leave the track for very short intervals only.

Having assimilated the available sensory information, the human body responds by use of its servomechanisms, which move the limbs in such a way that the car maintains the desired position on the track. Speed of response - the 'simple reaction time' - is of basic importance in the same way that inherent intelligence is of basic importance in the driver's psychological make-up, in that a low I.Q. is as incompatible with top class race driving as a slow 'reaction time'. Of far greater importance in race driving efficiency is the ability to memorise patterns of the car's behaviour and response to the controls. In the short term, this leads to a smooth flow of neuromuscular output in reply to previously 
experienced inputs: in a way, the driver's reflexes are then 'conditioned'. In the long term, this ability is a measure of racing experience and the benefit gained thereby, so that experienced drivers whose physiological systems are well past their theoretical maximum efficiency can compensate by being able to cope easily with situations which have nearly all been encountered in the past.

The Task

The race driver is confronted by continuous 'tracking tasks' of 'pursuit' and 'compensatory' type, plus intermittent 'setting' tasks such as changing gear. Driving the car round the circuit is a pursuit task, whereby the operator seeks to maintain, by movement of a control - the wheel - a desired position relative to a moving reference - the race track. Meanwhile, maintaining the longitudinal relationship of care and track is a compensatory tracking task, where control movements are being used to compensate for the forces which attempt to destroy this constant relationship.

The ultimate skill of the racing driver is directly related to the efficiency with which he performs these tracking tasks, thus fulfilling his part of the closed loop system of track/car/Man input/man output/car/ track. Can we, then assess this skill? At present, the only easy measurement is by the speed and consistency with which the driver laps a closed circuit. But it is well recognised that many apparently extremely fast drivers do not perform particularly well in races. To see why, further analysis of the tracking task is required. It is possible to build laboratory machines which can provide operators with pursuit or compensatory tracking tasks. By suitable instrumentation it is possible to measure the operator's performance in terms of his ability to cope accurately with given (or chosen) speeds, and in terms of the errors he makes. At an early stage in the development of such experiements many interesting lines of enquiry emerged, among them the varying patterns of movement of the controls chosen by different operators in striving for the fastest and most accurate performance possible. It is evident that, in a given racing car, two drivers will use the steering wheel differently while producing the same lap times: this is part of the driving 'style'. In the laboratory, many differences are found between groups of operators who favour fine, frequent control movement and groups preferring slower, coarser movements. There are predictable differences in effect; for instance, a rough ride in a harsh car on a bumpy track will decrease the ability of the latter group to a limited extent only, whereas the former group - potentially faster and neater - is more dependent on a stable platform to bring forth his very best performance. Here, then, we have a glimmering of just one factor which might let a given driver perform particulatly well in one class of racing, but never shine in another. 
Skill is a vague concept, hard to define, but can be expressed as a function of what it costs the man to achieve a given performance. For example, suppose that a champion driver and a promising newcomer are in close company at racing speed. At this speed, however fast, the champion holds some major advantages. He is using less of his available mental and physical resources, which enables him to apply more attention to race tactics, strategy, his environment and his instruments than his competitor - possibly already $100 \%$ extended - can manage. The stopwatch, recording identical lap times, is no measure of the cost to the man of maintaining these times. Can we, then, measure the cost of performance, rather than the performance itself? Applied psychologists at Farnborough and Cambridge are trying to answer this very question, by using the concept of a 'primary' and 'secondary' task to determine the amount of 'spare capacity' available, and thus the cost to the individual of the primary task concerned. In the laboratory, the primary task is generally one which most people can manage without too much difficulty. In this context, a tracking task would be most appropriate for the primary; the secondary task might be, for example, a light which has to be switched of $f$ as quickly as possible after it has flashed on at random intervals. The speed and efficiency with which the operator copes with this secondary task is a measure of his spare capacity, and thus of the ease with which he is dealing with the primary activity. A few drivers in the very top echelons have been noted for the way they can pick out faces in the crowd, and wave to photographer friends, with no decrement to their lap times: they are simply not being fully extended by their primary task of race driving.

But it is obviously impossible to apply similar research methods to hazardous occupations like the driving of racing cars; the risk is overtaxing a fully occupied racing driver is self-evident! Other estimates of capacity and bodily 'arousal' must be tried. Physical and mental stress affects the working of the body as manifest by hyperventilation, tachycardia, sweating and unconscious muscular tension. Indeed, the heart rate has long been taken as a measure of stress - many drivers in the RAC Rally had pulse counters strapped to their chests. But the pulse rate is only one measure; given the same stress, one man's heart may beat 140 times per minute, while his competitor's pulse is a steady 80 but with a skin wet with sweat. The problems inherent in full instrumentation of heart, respiration, nerve and muscle activity are too great to be tackled with any enthusiasm in a single seater, but more research on these lines in, say, a racing sports car would be of absorbing interest.

Skill can be defined in another way: the ability to function efficiently under stress. The stresses to which a racing driver may be exposed are very varied indeed, and this is not the time to expand on them in any detail. Physiologically, he may be subjected to excessive heat, vibration, noise and physical discomfort; psychologically, to fatigue, poor motivation and fear. The degree to which such stress is affecting the task can only be measured with any accuracy by the 'secondary task' method outlined above. How many times, for instance, have we seen an exhausted racing driver lifted out of his car; yet a few seconds earlier the same man was lapping whithin a few seconds of lap record. Clearly, his 
primary tracking task has been unaffected by the stress, but as this stress has taken up an increasing amount of his available spare capacity he has been drawing nearer and nearer the inevitable moment when the stress demands primary attention. At this point he will err, in speed or in accuracy. Meanwhile, his race planning has been suffering through his diminished spare capacity. The secondary task should never reduce the efficiency with which the primary task is performed; if it does, it usually means that the primary task has been poorly learned, or that the operator is poorly motivated. The novice who loses control while being lapped has, in effect, temporarily disregarded his primary task in order to cope with the secondary.

The job for which the professional racing driver is paid, and the ambition of all his competitors, is to win motor races and avoid 'accidents! Whatever may be the feeling regarding the relevance of increased safety in motor racing, the fact remains that no driver accrues any credit by regularly disappearing into the undergrowth, however fast. Grand Prix racing drivers have long been regarded with some awe - and justly - as artists, but perhaps by turning the spotlight of science on to the driver as well as on his machine the benefit will be shown by an increase in both safety and out-and-out speed.

\section{References}

Krikler, Berenice. Brit. J. Psychiat., 111, 192-194, 1965.

Taggart, Peter and Gibbons, David. Brit. Med. J., 1967, 1. 411-412

Collins, Vincent P., N.A.S.A. Report CR 570, 1966.

Benson, A.J., Huddlestone H.F., and Rolfe, J.M. Human Factors, 1965,7, 